\title{
Les paramètres traductologiques du transfert interlingual de l'humour
}

\author{
Magdalena SZEFLIŃSKA-BARAN \\ Marek BARAN \\ Université de Łódź
}

Resumen: Este artículo pretende introducir algunas consideraciones extralingüísticas en el campo interdisciplinario de la traducción del humor. Esencial para esta tarea es una breve introducción a la teoría más relevante de la traducción del humor: la adaptación de Attardo de la Teoría general del humor verbal para la traducción. Attardo ha agregado cuatro consideraciones extralingüísticas, que explican los factores que se encuentran fuera de los textos fuente y destino.

Palabras clave: humor verbal, traducción, perspectiva sociolingüística

\begin{abstract}
This paper intends to introduce some extralinguistic considerations to the interdisciplinary field of humour translation. Essential to this task is a brief introduction to the most relevant theory of humour translation: Attardo's adaptation of the General Theory of Verbal Humour for translation. Attardo has
\end{abstract}


added to this four extralinguistic considerations, which account for factors which lie outside both the source and target texts.

Keywords: verbal humour, translation, sociolinguistic approach

Les paramètres traductologiques du transfert de textes humoristiques se situent aussi bien du côté de leur compréhension que de celui de leur reproduction dans la langue-culture cible et même au stade de choix de textes à traduire. Dans sa théorie générale de I'humour verbal, Attardo (2002) énumère six facteurs linguistiques et extralinguistiques qui déterminent la traduction de textes humoristiques. Parmi ces facteurs, il y a des savoirs sur la langue donnée, sur la stratégie narrative appliquée, la situation présentée, le mécanisme logique qui, le cas échéant, sert de base pour la création de l'effet humoristique ainsi que sur l'opposition des isotopies et sur le contexte de départ et le public cible, au sens large des termes. Parmi ces derniers paramètres, nous retrouvons les cadres: temporel, géographique et social, liés à l'apparition d'un texte humoristique. Les conditions du transfert de l'humour verbal se présentent comme un éventail de facteurs hétérogènes parmi lesquels semblent prévaloir ceux qui appartiennent au domaine socioculturel contre les facteurs purement linguistiques.

Dans la phase du choix de textes à traduire, le rôle des paramètres extralinguistiques est primordial. Il paraît qu'uniquement l'humour à portée plus ou moins universelle se prête à la traduction ce qui exclut d'autres types d'humour : par exemple I'humour ethnique soumis aux limites géographiques ou bien l'humour politique déterminé du point de vue temporel par le principe d'actualité. Le manque du fond commun soit géographique soit socioculturel entre des communautés réduit la possibilité du transfert entre elles de textes humoristiques qui garderaient leur caractère et leur fonction. 
2019. "Les paramètres traductologiques du transfert interlingual de l'humour" Sabir. International Bulletin of Applied Linguistics, 1: 144-162

Les jugements parfois opposés, portés sur la traduisibilité de l'humour proviennent du caractère ressenti immédiatement comme doublement constitué de l'humour : caractère linguistique et caractère socioculturel. La combinaison dans des proportions variables de ces deux facteurs rend la traduction plus ou moins aisée. Cette ambivalence est déjà visible dans I'histoire de la notion d'humour. Escarpit (1960 : 37) en parlant de I'humour actif et passif, souligne que la différence entre un homme d'humour, qui renvoie ici à une attitude active, et un humoriste, qui représente une posture passive chez Morris (1744 : 13), cette distinction se grave dans la définition contemporaine de l'humour. C'est ainsi que l'humoriste, l'homme qui a le sens de I'humour et qui continue à vivre en Angleterre, représente un côté national de I'humour, constitue la partie intraduisible, inexportable du phénomène de I'humour, celle qui restera à jamais inaccessible aux étrangers. "Il est, à proprement parler, le produit du Réflexe anglais [...] ». (Escarpit, 1960 : 37) L'homme d'humour qui renvoie à I'humour esthétique, au contraire, est susceptible d'un langage universel, traduisible et transmissible entre de différentes nations. La notion d'humour qui cristallise au XVIII siècle, en gardant surtout la partie intellectuelle, consciente et esthétique plus proche de l'esprit, perd la vitalité du sens de l'humour.

Les poètes français, à la charnière du XIXème et XXème siècles voient dans I'humour un moyen d'exorciser les angoisses de l'homme social moderne. Escarpit cite Max Jacob, qui définit I'humour ainsi : «Une étincelle qui voile les émotions, répond sans répondre, ne blesse pas et amuse. ${ }^{1}$ Cette définition signifie la découverte du sens social de l'humour, non plus au niveau superficiel des automatismes, des réflexes, comme chez les Anglais, mais de manière créatrice comme une attitude face à la vie. Escarpit conclut en prétendant que l'humour moderne est sociologique ce qui semble trouver sa confirmation dans un rôle assez

\footnotetext{
${ }^{1}$ Escarpit (1960 : 69) cite la définition de I'humour de Max Jacob, prise de : Conseils à un jeune poète, p. 81.
} 
important joué par l'humour dans la communication d'aujourd'hui. Escarpit (op.cit. : 72) enferme le rôle social de l'humour dans la formulation suivante : «L'humour est I'unique remède qui dénoue les nerfs du monde sans l'endormir, lui donne sa liberté d'esprit sans le rendre fou et mette dans les mains des hommes, sans les écraser, le poids de leur propre destin. »

\section{La spécificité de la traduction de l'humour verbal}

Ce type d'humour ne pose pas de grands problèmes en traduction à condition de trouver le même type de décalage entre le contenu et le style. Dans cet énoncé, l'humour naît de la généralisation au niveau du contenu et du style de l'énonciation qui caractérise son expression.

Lorsque l'humour naît d'un double jeu sur la langue et sur la réalité à laquelle se réfèrent les mots, alors la traduction présente des difficultés parfois difficiles à surmonter. En effet si le présupposé est une connaissance de faits de civilisation, il faut que ces connaissances soient acquises au préalable.

La traduction de l'humour exclut une adjonction des explications, des notes de bas de page qui tuent l'effet de surprise et modifient la structure interne d'un texte humoristique et d'un échange comique qui possèdent leur propre dynamique. Cette dernière remarque s'avère particulièrement vraie pour le traitement en traduction des blagues politiques supposant des connaissances préalables. Pour que leur traduction soit fidèle, tout ce fond de connaissances doit être connue du locuteur de la langue-cible. Ainsi, la traduction de l'humour oscille-t-elle entre le transfert appauvri du contenu original, rendant uniquement compte des jeux de langue humoristiques de surface, et la traduction qui fournit aussi des allusions historiques, sociales, culturelles propres à une société donnée. Le même problème concerne les blagues ethniques nationales qui jouent sur des préjugés nationaux, supposés connus de la communauté. C'est ainsi que le schéma stéréotypé 
2019. "Les paramètres traductologiques du transfert interlingual de l'humour" Sabir. International Bulletin of Applied Linguistics, 1: 144-162

humoristique se répète sous forme de versions nationales : les histoires belges en France sont traduites par des histoires polonaises aux États-Unis et peuvent apparaître comme des histoires irlandaises en Grande-Bretagne ou des blagues russes en Pologne.

La théorie interprétative de la traduction (la théorie du sens) souligne également l'importance du savoir partagé pour la compréhension du sens du texte, ce qui constitue aussi une condition sine qua non de la compréhension et de la reproduction du discours humoristique. Dans le processus de construction du sens, le traducteur a connaissance de la situation de communication, qui inclut l'émetteur, le destinataire, le lieu, l'époque et la raison d'être du message. Les éléments non linguistiques qui contribue à la compréhension ne s'arrêtent pas là et, pour que la communication s'établisse vraiment, il faut que les interlocuteurs aient un certain savoir partagé, qui englobe une certaine connaissance commune du sujet du discours ou du texte. La saisie du sens par le lecteur/traducteur repose donc non seulement sur les significations linguistiques du message, mais aussi sur le recours à son bagage cognitif, antérieur à la réception du texte, à ses souvenirs ou aux associations d'idées suscitées en lui. Cela constitue une parenté claire avec la compréhension de la communication humoristique qui, n'ayant pas de but strictement informationnel et étant une forme indirecte de communication, construit son effet humoristique sur les éléments extralinguistiques communs pour une communauté linguistique. Le traducteur ajoute à ses connaissances de la situation et du sujet les éléments qui lui sont apportés par le contexte verbal et cognitif, c'est-à-dire par les composantes formelles et narratives de l'original. Dans la théorie interprétative de la traduction, toutes les connaissances préalables et concomitantes à la réception du texte qui entrent en jeu dans la compréhension du sens constituent un ensemble de savoir appelé les compléments cognitifs. 
Il serait bon de préciser, surtout dans le cas du discours humoristique, que la saisie du sens ne se situe pas uniquement sur le plan notionnel. En effet, la traduction ne vise pas seulement à rendre le contenu du texte ou discours donné, mais aussi son effet émotionnel, indissociable du premier. En effet, le traducteur ne perçoit pas consécutivement d'abord la dénotation, puis les nuances connotatives ou stylistiques. Le sens inclut donc le vouloir dire de l'auteur et, même dans un texte à visée pragmatique, son «comment dire ». Dans la communication humoristique, c'est justement ce « comment dire » qui prévaut sur « ce qui est dit », en imposant une interprétation du contenu dans l'optique humoristique qui le détermine. Seule la restitution combinée de ces deux niveaux peut garantir une bonne traduction. La traduction est une opération mentale dont l'objectif n'est pas de produire des correspondances linguistiques, mais des équivalences textuelles. Cela signifie qu'elle se situe non dans le domaine de l'identité en traduction, mais de l'analogie fonctionnelle et pragmatique. C'est dans cette optique de l'équivalence ou de l'analogie, du « comme », que se situe la traduction de textes humoristiques, ce qui passe inévitablement par l'interprétation et les choix personnels du traducteur. L'humour verbal peut être défini comme une attitude spécifique face à la réalité, exprimée à l'aide de moyens linguistiques et paralinguistiques propres à l'expression du comique au sein d'une communauté, dont la fonction primordiale est celle de régulateur social. Il s'ensuit que l'approche traductologique de l'humour doit obligatoirement tenir compte de son côté pragmatique et fonctionnel sans pourtant nier son aspect linguistique.

\section{La traduction de l'humour dans la perspective sociolinguistique - la théorie de la médiation appliquée à la traduction des histoires drôles}

L'humour en tant qu'un régulateur important des relations sociales, joue un rôle décisif de médiation dans la communication aussi bien dans le cadre officiel, 
institutionnel que familial. Le concept de médiation se fonde sur la notion d'intermédiaire et de lien. L'élément médiateur est celui qui s'interpose entre deux autres éléments en les modifiant pour aider ou faciliter les relations. Au sens très large du terme, tout peut être médiateur de quelque chose dans la communication. Dans le domaine du langage nous avons affaire à la médiation du sens dans le cas de la traduction, de la reformulation ou du code-switching (deux codes en contact dans le même échange communicatif), (Martin, 2002 : 311). Le dire humoristique peut remplacer un sens absent ou négocier le statut du contenu socioculturel dans un nouveau contexte. C'est le cas de la traduction des histoires drôles qui a pour but d'intégrer leur contenu dans la culture d'arrivée en gardant leur rôle pragmatique. L'humour verbal propose une médiation linguistique pour exprimer un sens ou transmettre une situation parfois difficile à accepter ou à comprendre, d'une manière atténuante, légère. Dans ce sens, le rôle de l'humour est de faire communiquer tout le monde dans une symbiose parfaite tant au sein d'une communauté qu'entre plusieurs sociétés. Le potentiel médiateur des histoires drôles se manifeste à plusieurs niveaux. Premièrement, I'humour dans ce type de texte permet d'approcher une réalité difficile ou choquante du point de vue cognitif ou émotionnel. C'est le rôle d'apprivoisement ou d'exorcisme. Il n'est pas étonnant que l'éventail de sujets traités dans les histoires drôles soit stéréotypé :

1. le premier groupe de sujets est constitué par des thèmes liés à l'existence de I'homme et son fonctionnement social : la naissance et la mort, les parents et les enfants, l'amour sous toutes ses formes: des relations entre l'homme et la femme, la vie de couple, le mariage, l'école :

Quelle est la peine infligée pour bigamie?

- Deux belles-mères. (Peigné, 2007 : 25) 


\section{Szeflińska-Baran y M. Baran}

2019. "Les paramètres traductologiques du transfert interlingual de l'humour" Sabir. International Bulletin of Applied Linguistics, 1: 144-162

2. la catégorie suivante est constituée par des groupes socioprofessionnels, ethniques et nationaux: les médecins, les journalistes, les soldats et les policiers, les garçons de café, les gens célèbres, les dirigeants et les hommes politiques, les Juifs, les montagnards, les nationalités voisines (les Russes en Pologne, les Belges en France) :

Dans un dîner, un médecin se trouve à table près d'un avocat. Et il lui dit :

- C'est terrible. Chaque fois, dans une soirée, que je dis que je suis médecin il y a toujours quelqu'un qui me raconte ses symptômes, qui demande mon diagnostic et même mon avis sur le traitement que lui a donné un autre médecin. Quand vous avez ce genre de problème, maître, vous faites quoi ?

- Et bien, en rentrant chez moi je leur envoie une note d'honoraires: Consultation au dîner d'hier soir : 100 euros. Ils ne recommencent pas.

- C'est une excellente idée, fait le médecin, c'est ce que je vais faire.

Et le lendemain il reçoit une lettre de l'avocat : Consultation au dîner d'hier soir : 100 euros.

(Peigné, $2007: 234$ )

3. le troisième type selon le critère thématique englobe les histoires drôles qui se construisent autour d'un personnage stéréotypé incarnant un trait de caractère ou une caractéristique intellectuelle: les blondes, les secrétaires, les ivrognes:

Un prédicateur fait son sermon sur les ravages de l'alcoolisme.

- Je vous le demande, mes frères, s'écrie-t-il, que peut-il y avoir de pire que la boisson?

Et dans le fond de l'église une voix fait :

- La soif.. $\quad$ (Peigné, $2007:$ 244) 


\section{Szeflińska-Baran y M. Baran}

2019. "Les paramètres traductologiques du transfert interlingual de l'humour" Sabir. International Bulletin of Applied Linguistics, 1: 144-162

Il faut souligner que le critère thématique mentionné ci-dessus participe à la distinction de l'histoire drôle comme macrosigne humoristique qui se caractérise par un très haut niveau de stéréotypisation du contenu et de même, se distingue d'entre d'autres formes humoristiques comme, par exemple : la satire ou l'anecdote. Cette dernière est toujours bien située dans le contexte d'énonciation dans lequel elle apparaît: elle est située dans l'espace et dans le temps et fait partie de l'expérience du locuteur. Quant à la satire politique ou de mœurs, elle est bien ancrée dans la réalité de l'époque et au moment où le référent disparaît ou n'est plus au centre d'intérêt, la satire perd son pouvoir humoristique. Pourtant, ce qui est intéressant c'est le fait que la persistance suffisamment longue de la réalité extralinguistique, faisant l'objet de la satire, crée dans la société une nouvelle expérience cognitive et affective durable, laquelle permet la transformation de la satire en histoire drôle par le fait de généraliser une expérience qui devient un stéréotype mental. C'est le cas des histoires drôles sur la PRL en Pologne ou le régime communiste en Russie, qui font encore aujourd'hui partie du patrimoine humoristique de ces pays tout en restant vivantes au niveau pragmatique : elles font encore rire. En voici un exemple :

Na posterunku MO skarży się okradziony przed chwilą mężczyzna :

- Jakiś cudzoziemiec ukradł mi zegarek.

- Cudzoziemiec ? Może Amerykanin?

- Nie-odpowiada poszkodowany.

- Anglik? A może Francuz?

- $\quad$ Ani Anglik, ani Francuz.

- $\quad$ A kto to mógł być ? - pyta dalej posterunkowy.

- Myślę, że Fin - mówi niepewnie okradziony.

- Obywatelu, Finów w Polsce nie ma ! Może Rosjanin ? 


\section{Szeflińska-Baran y M. Baran}

2019. "Les paramètres traductologiques du transfert interlingual de l'humour" Sabir. International Bulletin of Applied Linguistics, 1: 144-162

- Tak, ale to pan powiedział, panie posterunkowy.

\section{La notion de stéréotype}

Le comique verbal présent dans les histoires drôles est enraciné soit dans la forme du signe (dans le signifiant), soit dans son côté sémantique (le signifié) : dans sa signification systémique, ou bien dans la culture présente en tant que savoir partager mentalisé ou connoté dans la langue. L'humour verbal devient, donc pour nous une posture énonciative ayant comme point de départ une manière subjective et volontaire spécifique de percevoir la réalité, qui se manifeste dans la communication, entre autres sous la forme d'histoires drôles, celles-ci profitant au niveau de l'expression de la forme et du contenu de différents types de comique (comique situationnel, verbal). Le contenu stéréotypé des histoires drôles constitue une condition sine qua non de leur fonctionnement dans la communication langagière intra- et interculturelle. Le stéréotype dans notre perspective, peut être défini comme suit : « Jako części składowe wspólnej potocznej wiedzy określonej wspólnoty językowo-kulturowej stereotypy pozostają implicytne, istnieją w sposób ukryty, nie ujawnia się ich i nie wypowiada, [...]. Jest to tak długo komunikatywnie funkcjonalne, jak długo są one podzielane przez wszystkich uczestników interakcji. Ponieważ stereotypowe zasoby wiedzy, zróżnicowane kulturowo, nie są przekazywane wprost, ich niejawna obecność może być również źródłem nieporozumień albo niezrozumienia. (Quasthoff, 1998: 22). Dans le corpus d'histoires drôles analysé, extraites de recueils ou de sites internet consacrés à I'humour, les stéréotypes présentent des catégories thématiques qui organisent l'ensemble des histoires drôles. Ce sont par exemple catégories telles que : Vie de couple, Blondes, Sexe, Politique, Toto, O Jasiu, U lekarza, Chistes de Lepe, O Wąchocku, etc. Parmi ces catégories, il y en a celles qui stéréotypées dans le sens 


\section{Szeflińska-Baran y M. Baran}

2019. "Les paramètres traductologiques du transfert interlingual de l'humour" Sabir. International Bulletin of Applied Linguistics, 1: 144-162

de renvoyer aux sujets les plus souvent traités dans des histoires drôles, par exemple : Vie de couple, mais il en a d'autres, aussi, dont le statut est différent : ce sont les noms propres qui deviennent des catégories stéréotypées dans le discours humoristique, dont la fonction pragmatique consiste, entre autres, à signaler le passage du registre sérieux au registre humoristique (ce qui impose aux interlocuteurs ou lecteurs un mode d'interprétation convenable). Au niveau sémantique, Toto, Jaś, Wąchock, Lepe, La Blonde deviennent des catégories stéréotypées, des prototypes, respectivement, de l'enfant, du lieu, d'un type de mentalité ou de tel ou tel trait du caractère, qui fonctionnent comme stéréotypes humoristiques dont le contenu constitue un point de repère pour la création d'une histoire drôle. Dans ce cas-là, le passage d'une langue à l'autre ne constitue pas un problème pour la traduction parce que chaque culture possède dans son patrimoine humoristique un stéréotype de la naïveté enfantine, du lieu absurde, de la bêtise. Il suffit de garder la convention humoristique en trouvant un nom équivalent qui fasse naître une histoire drôle. Il est à souligner que ce type d'histoires drôles, constituent seulement une illustration, sous forme ludique, du contenu stéréotypé véhiculé par le signe de Toto ou d'autres mentionnés ci-dessus. En voici quelques exemples :

Comment meurent les neurones d'une blonde?

Seuls. (Peigné, $2007: 603$ )

Dans une pizzeria, une blonde commande une margarita. En la sortant du four, le pizzaïolo lui dit :

- Je vous la coupe en deux ou en quatre?

- Oh, en deux! fait la blonde. Je serais incapable de manger quatre parts ! (Peigné, $2007: 603$ ) 
2019. "Les paramètres traductologiques du transfert interlingual de I'humour" Sabir. International Bulletin of Applied Linguistics, 1: 144-162

La maitresse dit à Toto:

- Toto, tu n'as pas fait tes devoirs !... Peux-tu me dire pourquoi ?

- Des frissons, de la fièvre, des vomissements...

- Et tu as réussi à venir à l'école ?

- Ben oui... C'est papa qui avait ça!

- Ton papa?

- Oui... Il n'a pas voulu faire les devoirs, il a dit : "Tant pis si on a un zéro!" (Peigné, 2007 : 16)

Dans les cas comme ceux-ci, la langue-culture cible disposant des équivalents structuraux quant à la forme de l'histoire drôle et des stéréotypes équivalents, la traduction garde tous les paramètres formels et sémantiques du texte de départ, y compris ses fonctions pragmatiques.

\section{Les paramètres linguistiques de la traduction de l'humour verbal}

Parmi les paramètres, qui ont un poids incontestable dans la traduction de I'humour verbal, nous retrouvons ses caractéristiques inhérentes déjà mentionnées à plusieurs reprises, tout au long de notre analyse. Les théoriciens qui s'occupent de la problématique du transfert interlingual de l'humour y voient essentiellement deux catégories d'obstacles à la traduction, se laissant classer dans deux groupes : celui de paramètres subjectifs et celui de facteurs objectifs. La première catégorie renfermerait tous les facteurs liés au producteur et récepteur de l'humour et aux conditions de sa production/utilisation et perception/interprétation, c'est-à-dire : le type de mentalité, les stéréotypes, le sens de l'humour, le savoir socioculturel partagé, le profil psychologique et social des interlocuteurs, leur statut. Dans le groupe de données objectives, nous pouvons énumérer les facteurs qui 
2019. "Les paramètres traductologiques du transfert interlingual de l'humour" Sabir. International Bulletin of Applied Linguistics, 1: 144-162

représentent la langue comme source ou moyen d'expression de l'humour, et les conventions sociolinguistiques concernant la communication humoristique. Aux catégories proposées ci-dessus, nous pouvons aussi appliquer la taxonomie proposée par Misri (1990) qui regroupe différentes composantes communicatives qui sont en jeu dans la traduction des unités phraséologiques. Selon Misri, dans l'approche de la traduction de l'humour verbal, il faut définir ou identifier les éléments essentiels, constitutifs de l'humour d'un texte pour les rendre dans un texte cible. Misri souligne l'importance de quatre composantes communicatives de I'humour, en en qualifiant deux comme obligatoires et deux autres comme facultatives. Parmi les composantes obligatoires, nous pouvons distinguer :

- $\quad$ une composante informative qui se laisse identifier par le biais de la paraphrase,

- une composante hiérarchique liée au registre de langue utilisé,

- une composante collocative qui concerne le degré de cohésion des éléments de telle ou telle expression humoristique ou d'un jeu de mots,

- une composante de conformité qui concerne l'accord de l'expression avec l'usage langagier.

Il y a encore d'autres éléments qui entrent en jeu dans la traduction de I'humour des jeux de mots, facultatifs ou spécifiques qui font partie, avec les composantes obligatoires, de la charge communicative des expressions humoristiques à recréer dans la traduction.

La taxonomie de Misri, présentée ci-dessus, concerne surtout la traduction des expressions phraséologiques, mais elle a été utilisée aussi pour la classification des composantes communicatives de la traduction des jeux de mots, p.ex. par J.Górnikiewicz (2008). La similitude des approches appliquées à l'analyse communicative des expressions phraséologiques et celle qui peut servir à analyser les jeux de mots humoristiques, surtout dans la perspective traductologique ou 
comparatiste a été également soulignée par d'autres chercheurs. Ce point de vue paraît être justifié notamment par le statut linguistique et communicatif des deux phénomènes: les expressions idiomatiques et les jeux de mots humoristiques, parfois même les histoires drôles, constituent des macrosignes, des unités communicatives, dont l'organisation interne suit des mécanismes bien stables et qui fonctionnent dans la communication comme des unités de sens, des entités pragmatiques. Lipińska (2002) souligne que, pour ce type d'expressions linguistiques, on propose le plus souvent le procédé d'équivalence comme technique de traduction. L'équivalence selon l'acception proposée par Vinay et Darbelnet, occupe une place importante dans la pratique et la théorie de la traduction. Selon Vinay et Darbelnet, elle signifie une technique de traduction qui rend compte de la même situation que dans le texte de départ mais en faisant appel à une forme linguistique différente. Dans ce cas-là, le tertium comparationis est constitué par le contexte d'emploi.

Les théoriciens de la traduction qui s'occupent plus particulièrement $\mathrm{du}$ transfert de l'humour verbal distinguent quelques types d'équivalence recherchés dans la traduction de textes humoristiques :

- l'équivalence de la substance textuelle, substance du contenu, substance de l'expression

- l'équivalence tournée vers la finalité, une équivalence effective (efficiente), pragmatique (Guidère 2000).

L'équivalence peut être jugée par rapport aux critères d'exactitude et de fidélité à l'original, en référence au sens, à l'intention, au contexte de communication, à l'effet à obtenir.

L'évaluation de la traduction-produit du comique se fait dans l'optique de l'effet humoristique à obtenir chez le public cible, dans la culture d'arrivée plutôt 
que dans la perspective de l'équivalence purement formelle. Il semble que dans cette situation la pragmatique prévale sur le côté formel de l'énoncé humoristique.

\section{En guise de conclusion}

Dans l'optique que nous avons adoptée, celle de l'approche sociopragmatique, la traduction est vue comme un échange verbal, qu'il soit oral ou écrit, contribuant à une communauté de savoirs et qui fait, de même, partie de la communication. Jean Peeters (1999: 168) souligne que : «Que l'on parle avec son boulanger, que l'on rédige une thèse de linguistique, ou que l'on traduise de la littérature étrangère dans une organisation telle qu'une maison d'édition, on produit, de concert avec d'autres protagonistes, ou contribuables, un certain patrimoine. En ce sens, la traduction n'échappe pas aux contraintes sociolinguistiques de l'interlocution dans une même langue. La traduction est une communication. » La dernière constatation implique que la traduction de textes humoristiques soit également contrainte aux exigences sociolinguistiques de l'échange interlingual. On pourrait remarquer que la traduction ne se limite pas au simple transfert de message et de sens, ni dans sa pratique ni dans son épistémologie, ce qui est d'ailleurs particulièrement vrai dans le cas de la traduction de I'humour. La traduction de I'humour semble être doublement ancrée dans l'interaction, analysée du point de vue linguistique et social, d'abord par sa matière, I'humour, et ensuite par le caractère du processus même de la traduction qui assume pleinement la fonction d'un acte social de communication. La notion de sens et celle de message ne sont pas suffisantes pour expliquer la traduction, il est nécessaire de prendre en considération des facteurs d'ordre sociolinguistiques, car : « [...], on traduit des façons socialement données de dire; on passe d'un message d'une société à un message d'une autre société, c'est-à-dire d'un usage linguistique 


\section{Szeflińska-Baran y M. Baran}

2019. "Les paramètres traductologiques du transfert interlingual de l'humour" Sabir. International Bulletin of Applied Linguistics, 1: 144-162

à un autre usage linguistique. »(Peeters, $1999: 168)$. Dans l'échange nous avons affaire à une structure logique sous-jacente qui détermine aussi bien sa production que sa réception et son interprétation. Cette idiomatisation de l'interaction concerne au même degré la communication humoristique. En effet, quand on parle de structures globales (dans la terminologie de Pierre Bourdieu) ou d'utilisations du cadre spatial (selon Kerbrat-Orecchioni), on veut souligner le fait que l'interaction au sens général ou celle à caractère humoristique ne se réduit pas à ce qui est de prime abord visible.

\section{Références citées}

Aladro, E.

2002. "El humor como medio cognitivo" en CIC : Cuadernos de Información y Comunicación , 7: 317-327.

Attardo, S.

1994. "Linguistic Theories of Humor" en V. Raskin, Mahadev Apte eds, Humor Research 1. Berlin - New York: Mouton/De Gruyter.

1997. "The semantic foundations of cognitive theories of humor ". Humor, 10 , 4: 395-420.

2001. "Humorous Texts. A Semantic and Pragmatic Analysis" en Raskin, V., Ruch, W. (eds.) Humor Research.

2002. "L'analyse des textes humoristiques" en Mongi Madini, 2000 ans de Rire. Permanence et Modernité, Coll. Linguistique et sémiotique, 42. París: Presses Universitaires Franc-Comtoises, 273-280.

2002. Translation and Humour: An Approach Based on the General Theory of Verbal Humour (GTVH). Manchester: St. Jerome Publishing. 


\section{Szeflińska-Baran y M. Baran}

2019. "Les paramètres traductologiques du transfert interlingual de l'humour"

Sabir. International Bulletin of Applied Linguistics, 1: 144-162

y V. Raskin.

1991. "Script theory revis(it)ed: Joke similarity and Joke representation model". Humor, 4, 3/4, pp. 293-347.

BOURDIEU, P.

1980. Le sens pratique. Paris: Éditions de Minuit.

1984. Questions de sociologie. Paris: Éditions de Minuit.

Émelina, J.

1996. Le comique. Essai d'interprétation générale. Paris : SEDES.

Emerson, J. P.

1969. "Negotiating the serious import of humor". Sociometry, 32, 2: 169-181.

Escarpit, R.

1960, 1967,1994. L'Humour, Coll. «Que sais-je ? », no 877, Paris, : P.U.F., 4 édition.

1976. Théorie générale de / information et de communication. Paris : Hachette.

Górnikiewicz, J.

2008. "Humor - strategie tłumaczeniowe (na przykładzie gier słów w filmie Alaina Chabata Astérix \& Obélix: Mission (léopâtre)". Konieczna-Twardzikowa et. M. Filipowicz-Rudek (réd.), Między Oryginałem a Przekładem XIII. Poczucie humoru a przekład, Kraków, 91-111.

Guidere, M.

2000. Publicité et traduction. Montréal : L'Harmattan.

Kerbrat-OrecchionI, C.

1980. L'énonciation de la subjectivité dans le langage. Partis : Armand Colin.

1981. "Comment comprend-on l'implicite". Comprendre le langage, Paris : Didier.

1986. L'implicite. Paris: Armand Colin.

1994. Les interactions verbales, t. III. Paris : Armand Colin. 


\section{Szeflińska-Baran y M. Baran}

2019. "Les paramètres traductologiques du transfert interlingual de l'humour"

Sabir. International Bulletin of Applied Linguistics, 1: 144-162

1998. "Gestion des conflits en communication de coalitions dans les polylogues". Symposium International sur l'Analyse de Discours, Madrid.

2005. Le discours en interaction. Paris : Armand Colin.

Lipińska, M.

2002. "La binarité des proverbes polonais et français dans l'optique traductologique". Kacprzak, A. (réd.), Points communs: linguistique, traductologie, glottodidactique, Wydawnictwo Biblioteka, Łódź, 165-179.

Martin, G.-V.

2002. "Humour et médiation: structure médiative et figure médiative". Mongi Madini, 2000 ans de Rire. Permanence et modernité, Besançon, 311-320.

Misri, G.

1990). "La traductologie des expressions figées". Etudes traductologiques. Paris Minard Lettres Modernes.

Peeters, J.

1999. La médiation de l'étranger. Une sociolinguistique de la traduction, Coll. Traductologie. Artois Presses Université.

Quasthoff, U. M.

1998. " Etnocentryczne przetwarzanie informacji. Ambiwalencja funkcji stereotypów w komunikacji międzykulturowej". Anusiewicz, J., Bartmiński, J. (réd.), Język a kultura. Stereotyp jako przedmiot lingwistyki. Teoria, metodologia, analizy empiryczne, Towarzystwo Przyjaciół Polonistyki Wrocławskiej, Wrocław, 11-30. Vinay, J.-P. et J. Darbelnet.

1977. Stylistique comparée du français et de l'anglais. Méthode de traduction. Paris : Didier. 
M. Szeflińska-Baran y M. Baran

2019. "Les paramètres traductologiques du transfert interlingual de l'humour" Sabir. International Bulletin of Applied Linguistics, 1: 144-162 